\title{
Radiation patterns of multi-moded corrugated horns for far-IR space applications
}

\author{
J. Anthony Murphy ${ }^{\mathrm{a}, *}$, Ruth Colgan ${ }^{\mathrm{a}}$, Creidhe O’Sullivan ${ }^{\mathrm{a}}$, Bruno Maffei ${ }^{\mathrm{b}}$, \\ Peter Ade ${ }^{b}$ \\ a Department of Experimental Physics, National University of Ireland Maynooth, Co. Kildare, Ireland \\ ${ }^{\mathrm{b}}$ Queen Mary and Westfield College, London E1 4NS, UK
}

Received 5 September 2000

\begin{abstract}
Multi-moded horn antennas are now being proposed for far-IR space imaging systems in which diffraction limited resolution is not required (e.g. the High-Frequency Instrument (HFI) on the ESA PLANCK Surveyor). In such systems individual modes in the waveguide filter section feeding the horn can couple independently to an overmoded detector (such as a bolometer in an integrating cavity). The number of modes is chosen to optimize the coupling efficiency to the source without compromising any spillover losses. We consider in detail the case of a cylindrically symmetric corrugated configuration, presenting two alternative techniques for modelling such few-moded systems. The first approach is based on a mode-matching description of propagation in a non-uniform waveguide structure, while the second approach makes use of hybrid mode solutions for a waveguide with corrugated walls assuming a uniform but non-isotropic impedance. We present practical examples comparing the radiation patterns predicted by both models. (C) 2001 Elsevier Science B.V. All rights reserved.
\end{abstract}

PACS: $84.40 . \mathrm{Ba} ; 95.85 . \mathrm{Bh} ; 95.85 . \mathrm{Gn}$

Keywords: Multi-moded horn antennas; Bolometric detectors; Far-IR

\section{Introduction}

Few-moded corrugated horn antennas are now being proposed as feeds for imaging arrays in a number of far-IR astronomical receiver systems, such as, for example, the two highest frequency channels on the European Space Agency PLANCK Surveyor satellite [1]. In such cases, where dif-

\footnotetext{
${ }^{*}$ Corresponding author. Tel.: +353-1-708-3771; fax: +353-1708-3313.

E-mail address: anthony.murphy@may.ie (J.A. Murphy).
}

fraction limited resolution is not required, overmoded horns offer the possibility of a high level of coupling efficiency to a broadband source. Furthermore, corrugated horns have a low sidelobe response pattern, thus minimizing at the telescope the spillover which can couple in background noise. In the case of diffraction limited resolution a single-moded horn can be well matched to the telescope aperture for maximum on-axis gain. However, if a lower resolution is set for the system, the optimum number of modes is best determined by maximizing the coupling efficiency to the source field, given the constraint of an acceptable 
spillover or edge-taper level at the telescope (or at a pupil stop if one is present in the front-end optics). In effect the design goal in an imaging array is optimum coupling to a source whose extent is equal to the degraded resolution of the system. It is worth noting that only some of the higher-order modes affect the on-axis gain significantly, while others have a determining effect on the overall beamwidth.

For good control of the radiation pattern we assume the horn is connected to an integrating cavity containing an incoherent detector via a waveguide filter which limits the number of modes that can propagate (see Fig. 1). We also assume a flared section at the back of the waveguide (i.e. a back-to-back horn configuration), so that there is good coupling without an impedance mismatch to an integrating cavity. A sudden step in impedance would give rise to reflections reducing the coupling efficiency to a broadband source and affecting the beam pattern of the horn antenna. In this configuration the incoherent detector independently couples to the individual spatially coherent modes in the flared waveguide section at the cavity end. Only modes above cut-off (non-evanescent) propagate through the filter, of course, and there will be scattering of power between modes if the waveguide is not of uniform cross-section (as for example if the guide is corrugated).

The calculation of the far-field radiation pattern of a multi-moded horn amounts to summing in quadrature (i.e. in intensity) the radiation patterns of all non-evanescent modes at the entrance to the system (cavity end of the back-to-back horn/ waveguide structure) [2]. We can see this from the following argument. The radiation pattern of a

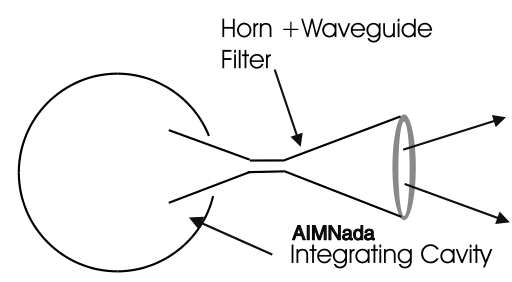

Fig. 1. Sketch of the optical system considered in the paper consisting of a corrugated horn and waveguide filter feeding an integrating cavity. multi-moded horn is determined by calculating the power coupling between a far-field point source of constant intensity at position $(\theta, \phi)$ and the absorbing cavity. Consider all possible waveguide modes $\psi_{n}$ than can exist at the aperture of the waveguide flare. Each of these $\psi_{n}$ when propagated through the guide and horn has a far-field radiation pattern given by $P_{n}(\theta, \phi)$, which represents the power coupling of the mode to a point source in the direction $(\theta, \phi)$. A particular modal field $\psi_{n^{\prime}}$ is transmitted to the horn aperture with an efficiency of $\eta_{n^{\prime}}$, which will only be non-zero for a finite number of modes $N$. If the $\psi_{n}$ at the flare aperture are true eigenmodes of the horn and guide, then $\eta_{n}$ is unity for those modes that are not cut off by the guide, otherwise $\eta_{n}$ is negligibly small. If the $\psi_{n}$ are not true eigenmodes, they will be scattered as they propagate but with each $\psi_{n}$ still mapping onto a spatially coherent field $\phi_{n}$ at the horn aperture (producing a radiation pattern given by $\left.P_{n}(\theta, \phi)\right)$. Provided there are no reflections the detector in the black body cavity can absorb the all of the power coupled to a particular $\psi_{n^{\prime}}$ modal channel independently of all the other $\psi_{n}$ channels without any interference (which would not be the case for a coherent detector). The total power coupled from the point source to the cavity by all of the modes in the horn aperture is then given by $P_{\text {total }}(\theta, \phi)=\sum_{n} P_{n}(\theta, \phi)$.

Using standard techniques borrowed from antenna engineering it is possible to model such partially coherent multi-moded horns in a straightforward way $[3,4]$. We present two different approaches. The first of these, known as the modematching technique, is based on regarding the structure as a sequence of smooth walled cylindrical waveguide sections each of which can support the appropriate set of TE and TM modes. Thus, the longitudinal cross-section of the waveguide and horn structure is viewed as a series of steps. At each corrugation there is a sudden change in the radius of the guide with a resultant scattering of power between the modes. In order to predict the effect of such scattering the total electric and magnetic fields are matched across the waveguide step so that conservation of complex power is maintained. Extending the technique leads to the scattering matrix description of 
propagation in the complete waveguide and horn structure as discussed in detail by Olver et al. [5]. Note that only azimuthal order $n=1$ tends to be of interest for horns connected to coherent detectors mounted in fundamentally moded waveguides. In this paper, in which we have to deal with incoherent detectors, we have extended the technique to higher values of $n$. By following the propagation all the way to the horn aperture the mode-matching technique can be used to predict the radiation patterns of corrugated horn antenna feeds. The technique is especially useful if the number of corrugations per wavelength is not very large, or the horn shape is profiled rather than being a simple cone. In Section 2 of this paper we discuss modal-matching modelling as applied to multi-moded far-IR horn antennas.

The second approach to modelling propagation in corrugated structures is based on the uniform surface impedance approximation. A hybrid mode model of wave propagation in a corrugated waveguide can be developed based on the assumption of many corrugations per wavelength and no sudden change in the waveguide or horn profile. The corrugated wall of the guide is regarded as having a uniform non-isotropic surface impedance (with a zero azimuthal transverse component and non-zero longitudinal component determined by the depth of the corrugation slots). In general the longitudinal components of both the electric and magnetic fields are required to satisfy the boundary conditions. The true modes of propagation of such a system cannot be pure TE or TM, but rather a hybrid combination that depends on the depths of the corrugations and diameter of the guide. The application of the boundary conditions determines the modal propagation coefficients and transverse wave numbers of a complete set of such hybrid modes. The surfaceimpedance method is simple and reasonably accurate and is particularly useful in the design of the waveguide filter section. However, for high accuracy the effects of space harmonics in the corrugation slots should be included. This will be necessary if the guide dimensions become too large or the slot widths are greater than $\lambda / 2$ [6].

The advantage of the hybrid mode model is that computationally it is much more efficient than the mode-matching approach, since hybrid modes are true eigenfunctions of the propagation transform and no scattering is involved. The modes can be regarded as propagating into the conical horn and developing a spherical wavefront which is equal to the slant length of the horn at the horn aperture. It is relatively straightforward to predict mode cutoff effects in the waveguide sections in terms of the waveguide radius and the slot depths. To achieve the same results using the modal-matching approach would require a tedious computationally intensive investigation of the parameter space in question. In Section 3 we present the hybrid mode model in more detail as applied to multi-moded waveguides and horn antennas.

In Section 4 we discuss some practical issues.An example is then analysed using the approaches outlined in Sections 2 and 3, the performance of the filter being predicted by the hybrid mode model while the final radiation pattern presented assumes the superior accuracy of the mode-matching model.

\section{Modal-matching model}

In the modal-matching technique the corrugated horn/waveguide structure is regarded as a sequence of cylindrical waveguide segments with the radius stepping between the top and bottom of the corrugation slots. For each segment the natural modes of propagation are the usual TE and TM modes of a uniform cylindrical waveguide. At the interface between two segments there is a sudden change in the guide radius and the power carried by the individual modes will be scattered between the backward propagating modes in the first guide segment and the forward propagating modes in the second guide. This results, for a corrugated structure with many such steps, in both forward and backward propagating fields existing within each segment. The modal-matching technique is based on matching the total transverse field in the two guides at the junction (expressed as a sum of both forward and backward propagating modes) so that complex power is conserved and the usual boundary conditions apply to the fields at the conducting walls. 
The mode scattering properties of such an interface between two guides can be represented by a scattering matrix $S$, in which the reflection and transmission characteristics are determined by the usual equation (see for example Ref. [5]):

$$
\left[\begin{array}{l}
{[B]} \\
{[D]}
\end{array}\right]=[S]\left[\begin{array}{l}
{[A]} \\
{[C]}
\end{array}\right]=\left[\begin{array}{ll}
{\left[S_{11}\right]} & {\left[S_{12}\right]} \\
{\left[S_{21}\right]} & {\left[S_{22}\right]}
\end{array}\right]\left[\begin{array}{l}
{[A]} \\
{[C]}
\end{array}\right]
$$

$A$ and $B$ are vectors made up of the forward and reflected mode coefficients, respectively, looking into the system at the input side, and $C$ and $D$ are vectors of the incident and transmitted mode coefficients, respectively, looking into the system at the output side. $S_{11}$ etc. are all matrices theoretically of infinite size but obviously for computational purposes truncated to as small a number of modes as is necessary for high accuracy.

For cylindrical waveguides the transverse electric fields of the two orthogonal sets of TM modes (distinguished by superscripts $\mathrm{c}$ and s) are given by:

$$
\begin{aligned}
& \left(\begin{array}{c}
\mathbf{e}_{n l}^{\mathrm{TM}, \mathrm{c}} \\
\mathbf{e}_{n l}^{\mathrm{TM}, \mathrm{s}}
\end{array}\right)=\sqrt{\frac{\left(2-\delta_{n 0}\right)}{\pi a^{2} J_{n+1}^{2}\left(p_{n l}\right)}}\left[J_{n}^{\prime}\left(p_{n l} r / a\right)\left(\begin{array}{c}
\cos n \phi \\
\sin n \phi
\end{array}\right) \hat{\mathbf{r}}\right. \\
& \left.+\frac{n J_{n}\left(p_{n l} r / a\right)}{p_{n l} r / a}\left(\begin{array}{c}
-\sin n \phi \\
\cos n \phi
\end{array}\right) \hat{\phi}\right],
\end{aligned}
$$

where $p_{n l}$ represents the $l$ th zero of $J_{n}(z)$ and $a$ is the guide radius [7]. The two possible orthogonal modes for each value of $n$ and $l$ arise from the choice of the longitudinal $z$-component of the appropriate field being either proportional to $\cos (n \phi)$ or $\sin (n \phi)$ - hence the use of the two superscripts $\mathrm{c}$ and $\mathrm{s}$ to represent the two corresponding cases. The transverse electric fields of the corresponding two sets of TE modes are:

$$
\begin{aligned}
& \left(\begin{array}{c}
\mathbf{e}_{n l}^{\mathrm{TE}, \mathrm{c}} \\
\mathbf{e}_{n l}^{\mathrm{TE}, \mathrm{s}}
\end{array}\right)=\sqrt{\frac{\left(2-\delta_{n 0}\right)}{\pi a^{2}\left(1-\left(n / q_{n l}\right)^{2}\right) J_{n}^{2}\left(q_{n l}\right)}} \\
& \times\left[\frac{n J_{n}\left(q_{n l} r / a\right)}{q_{n l} r / a}\left(\begin{array}{c}
\cos n \phi \\
-\sin n \phi
\end{array}\right) \hat{\mathbf{r}}\right. \\
& \left.-J_{n}^{\prime}\left(q_{n l} r / a\right)\left(\begin{array}{c}
\sin n \phi \\
\cos n \phi
\end{array}\right) \hat{\phi}\right] \text {, }
\end{aligned}
$$

where $q_{n l}$ represents the lth zero of $J_{n}^{\prime}(z)$ [7]. The constant of proportionality has been chosen to make $\int_{A}\left|\mathbf{e}_{n l}^{\mathrm{TE} / \mathrm{TM}}\right|^{2} r \mathrm{~d} r \mathrm{~d} \phi$ equal to unity. On ordering the guide modes $\mathbf{e}_{n i}^{\mathrm{G}}$ so that those of odd order are $\mathrm{TE}_{n l}$ while those of even order are $\mathrm{TM}_{n l}$ any coherent field propagating within the waveguide filter and horn can be represented by the expression:

$$
\begin{aligned}
\mathbf{e}_{\text {total }} & =\sum_{n l} \alpha_{n l}^{\mathrm{c}} \mathbf{e}_{n l}^{\mathrm{TE}, \mathrm{c}}+\alpha_{n l}^{\mathrm{s}} \mathbf{e}_{n l}^{\mathrm{TE}, \mathrm{s}}+\beta_{n l}^{\mathrm{c}} \mathbf{e}_{n l}^{\mathrm{TM}, \mathrm{c}}+\beta_{n l}^{\mathrm{s}} \mathbf{e}_{n l}^{\mathrm{TM}, \mathrm{s}} \\
& =\sum_{n i} A_{n i}^{\mathrm{c}} \mathbf{e}_{n i}^{\mathrm{G}, \mathrm{c}}+A_{n i}^{\mathrm{s}} \mathbf{e}_{n i}^{\mathrm{G}, \mathrm{s}},
\end{aligned}
$$

where $\mathbf{e}_{n, 2 l-1}^{\mathrm{G}}=\mathbf{e}_{n, l}^{\mathrm{TE}}$ and $\mathbf{e}_{n, 2 l}^{\mathrm{G}}=\mathbf{e}_{n, l}^{\mathrm{TM}}$.

In propagating through the waveguide and horn only scattering between modes of the same azimuthal index and same $z$-component dependance on $\cos (n \phi) / \sin (n \phi)$ is possible because of the cylindrical symmetry of the junction discontinuity. It is therefore only necessary to compute the scattering matrices $S^{(n)}$ for each azimuthal order separately. The $S^{(n)}$ are the same for both orthogonal mode sets. Note the $S^{(n)}$ must be calculated for all those values of $n$ for which modes can propagate. In the case of a coherent single mode horn only $S^{(1)}$ normally needs to be evaluated since only the $\mathrm{TE}_{11}$ and $\mathrm{TM}_{11}$ modes are non-evanescent in the waveguide filter section. Because of the azimuthal symmetry only scattering to $\mathrm{TE}_{1 n} / \mathrm{TM}_{1 n}$ modes is possible. Multi-moded horn calculations are therefore more complicated and computationally intensive.

At the step discontinuity where the guide radius increases from $a$ to $b$ (see Fig. 2) the submatrix elements of the scattering matrix $S^{(n)}$ can be written as:

$$
\begin{aligned}
& S_{11}=\left(R^{*}+P^{+} Q^{-1} P\right)^{-1}\left(R^{*}-P^{+} Q^{-1} P\right), \\
& S_{12}=2\left(R^{*}+P^{+} Q^{-1} P\right)^{-1} P^{+},
\end{aligned}
$$

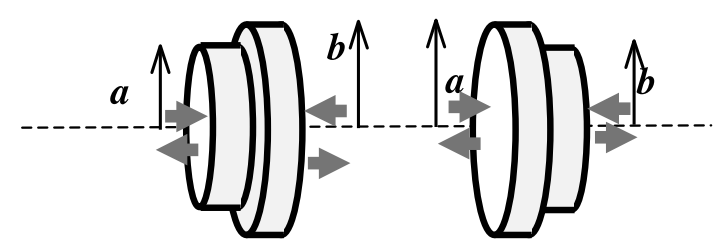

Fig. 2. Step discontinuity in guide where either $a>b$ or $b>a$. 
$S_{21}=2\left(Q+P\left(R^{*}\right)^{-1} P^{+}\right)^{-1} P$,

$S_{22}=-\left(Q+P\left(R^{*}\right)^{-1} P^{+}\right)^{-1}\left(Q-P\left(R^{*}\right)^{-1} P^{+}\right)$,

where for both the TE and TM modes defined above $R_{i j}=\left(1 / Z_{i}^{a}\right)^{*} \delta_{i j}$, and $Q_{i j}=\left(1 / Z_{i}^{b}\right)^{*} \delta_{i j}$ and crosscoupled powers $P_{i j}=\int_{0}^{2 \pi} \int_{0}^{a} \mathbf{e}_{n l}^{a}\left(\mathbf{h}_{n l^{\prime}}^{b}\right)^{*} r \mathrm{~d} r \mathrm{~d} \phi$, with appropriate values for the guide impedance $Z^{a / b}$ depending on the mode and guide radius [5], and $i$, $j$ are determined by the ordering of the $\mathrm{TE}_{n l} / \mathrm{TM}_{n l}$ modes $(i, j=2 l-1$ or 21 , respectively). Note the equations here differ slightly from those presented in Ref. [5]. The superscripts $a$ and $b$ on the impedance $Z$, and the $\mathbf{e}$ and $\mathbf{h}$ fields indicate the appropriate guide radius for the mode.

Assuming the modal field definitions above, the $P_{i j}$ can be evaluated from the following expressions for the cross-coupled power between the modes on either side of the discontinuity:

$$
\begin{aligned}
& P_{\mathrm{TE}_{n l} \rightarrow \mathrm{TE}_{n l^{\prime}}} \\
& =-\frac{\pi\left(1+\delta_{n 0}\right) D_{n l}(a) D_{n l^{\prime}}(b) q_{n l} J_{n}\left(q_{n l}\right) J_{n}^{\prime}\left(q_{n l^{\prime}} a / b\right)}{\left[\left(q_{n l^{\prime}} / b\right)^{2}-\left(q_{n l} / a\right)^{2}\right]\left(Z_{\mathrm{TE}}^{b}\right)^{*}},
\end{aligned}
$$

$$
\begin{aligned}
& P_{\mathrm{TM}_{n l} \rightarrow \mathrm{TM}_{n l^{\prime}}} \\
& =\frac{\pi\left(1+\delta_{n 0}\right) C_{n l}(a) C_{n l^{\prime}}(b) p_{n l^{\prime}} a / b J_{n}^{\prime}\left(p_{n l}\right) J_{n}\left(p_{n l^{\prime}} a / b\right)}{\left[\left(p_{n l^{\prime}} / b\right)^{2}-\left(p_{n l} / a\right)^{2}\right]\left(Z_{\mathrm{TM}}^{b}\right)^{*}}, \\
& P_{\mathrm{TE}_{n l} \rightarrow \mathrm{TM}_{n l^{\prime}}}=\frac{\pi D_{n l}(a) C_{n l^{\prime}}(b) a b n J_{n}\left(p_{n l^{\prime}} a / b\right) J_{n}\left(q_{n l^{\prime}}\right)}{\left[p_{n l^{\prime}} q_{n l}\right]\left(Z_{\mathrm{TM}}^{b}\right)^{*}},
\end{aligned}
$$

and $P_{\mathrm{TM}_{n l} \rightarrow \mathrm{TE}_{n l^{\prime}}}=0$, where

$$
C_{n l}(a)=\sqrt{\frac{\left(2-\delta_{n 0}\right)}{\pi a^{2} J_{n+1}^{2}\left(p_{n l}\right)}}
$$

and

$$
D_{n l}(a)=\sqrt{\frac{\left(2-\delta_{n 0}\right)}{\pi a^{2}\left(1-\left(n / q_{n l}\right)^{2}\right) J_{n}^{2}\left(q_{n l}\right)}} .
$$

As stated in Ref. [4] for a step in which $a>b$ (see Fig. 2) the appropriate scattering matrix subelements are $S_{11}(a>b)=S_{22}(a<b), S_{12}(a>b)=$ $S_{21}(a<b), S_{21}(a>b)=S_{12}(a<b)$, and $S_{22}(a>$ $b)=S_{11}(a<b)$. For a given azimuthal order the overall scattering matrix for the horn and wave- guide sections can be derived by cascading the scattering matrices for the sections in the appropriate way and including the phase delay effects of propagation along the length of each guide segment. At the end of the horn the fields are launched into free space. There is a sudden jump in the impedance and there may be significant reflections at that point. We can model this effect to a good approximation by assuming that the horn aperture lies in an infinite ground plane. This in turn can be modelled by a very large step into a waveguide of infinite diameter. Finite element analysis can be applied to the real horn aperture configuration, if low level sidelobe effects are important or if the aperture is very narrow in terms of the wavelength.

The modal fields at the horn aperture then propagate without scattering to the far field. If the waveguide filter is well matched to an integrating cavity by the use of a flared section, for example, all of the waveguide modes at the cavity entrance to the waveguide horn structure are assumed to be equally excited (i.e. carry the same amount of power). Note that the modes as defined above are not normalized for unity power. For each mode $n j$ excited at the entrance port there is a corresponding far-field radiation pattern $\mathbf{e}_{n j}$ produced by the horn:

$\mathbf{e}_{n j}^{\mathrm{c} / \mathrm{s}} \propto \alpha_{n j} \sum_{i}\left[S_{21}^{(n)}\right]_{i j} \mathbf{e}_{n i}^{\mathrm{ff} \mathrm{c} / \mathrm{s}}$,

depending on which of the orthogonal pair of modes is excited and $\mathbf{e}_{n i}^{\mathrm{ff}} \mathrm{c} / \mathrm{s}$ represents the radiation pattern of the modal field $\mathbf{e}_{n i}^{\mathrm{Gc} / \mathrm{s}}$ at the horn aperture. Note in the above sum the subscripts $n$ refers to the azimuthal order of the mode, and for $i$ and $j$ odd/even the modes are TE/TM, respectively. $\alpha_{n j}$ is the normalization factor which ensures the mode carries unit power at the entrance port. Since there is assumed to be no phase relationship between the modes at the entrance port of the system, the total far-field radiation pattern for the horn plus waveguide will be determined by summing the $\mathbf{e}_{n j}$ in quadrature. The far-field radiation pattern in intensity is then given by:

$$
P_{\text {total }}(\theta) \propto \sum_{n j}\left(\left|\mathbf{e}_{n j}^{\mathrm{c}}\right|^{2}+\left|\mathbf{e}_{n j}^{\mathrm{s}}\right|^{2}\right) .
$$


Some simplification is also possible in the analytical expression for $P_{\text {total }}$ as it clearly cannot depend on azimuthal angle because of the cylindrical symmetry. Applying the mode-matching approach in the design and analysis of multi-moded horn antennas is discussed in Section 4.

\section{Surface impedance model}

In the case of the alternative surface impedance model the horn and waveguide are considered as having their corrugated surface replaced by a wall of uniform non-isotropic impedance (with different impedances in the transverse and axial directions [6]). This average surface impedance clearly assumes many corrugations $(>3)$ per wavelength. It is extremely challenging to manufacture such horns at wavelengths shorter than about $350 \mu \mathrm{m}$ $(\sim 1.0 \mathrm{THz})$. Beyond this limit horns with few corrugations per wavelength cannot be reliably modelled by the surface impedance approach. The characteristics of the true hybrid HE/EH modes of propagation depend on the depths of the corrugations and the radius of the guide. The fundamental mode in a very wide cylindrical waveguide with corrugation slot depths of $\lambda / 4$ is the balanced hybrid $\mathrm{HE}_{11}$ mode. This is the modal field usually assumed at a corrugated horn aperture in predicting the radiation patterns for single-moded (coherent) systems. The effective impedance for any currents flowing across the corrugations is infinite, so that $H_{\phi}$ is zero. $E_{\phi}$ is also zero if we can assume there are many corrugations per wavelength. Similarly in a multi-moded horn we obtain higher-order balanced hybrid modes, where all such modes have zero transverse fields at the horn aperture edge. Consequently, such horns will produce radiation patterns with low sidelobe levels because of the gradually tapered fields at the horn aperture. Away from the balanced-hybrid condition (for slot depths $\neq \lambda / 4$ ) higher sidelobe levels are to be expected as the effective surface impedance across the corrugations is no longer infinite and non-zero fields exist at the corrugated walls. This may be an issue for the design of broadband horn feed systems. In the throat of a horn the effective impedance produced by the corrugations becomes mode dependent and is also non-infinite for slot depths of $\lambda / 4$. However, it is not necessary to maintain the balanced mode condition in the waveguide at the back of the horn as long as one knows the propagation characteristics there.

The critical issue for a multi-moded horn is the mode filtering properties of this waveguide section. In order to determine which modes can propagate in a corrugated waveguide it is necessary to solve the characteristic equation for the propagation coefficient $\beta$ (i.e. the guide wave number). Assuming there are several corrugations per wavelength the slots are narrow and only a single non-propagating TM mode is capable of existing in them [6]. Matching the admittance of the $m$ th order TM mode in the slot with that of the mode in the inner region of the guide (see Fig. 3) yields the characteristic equation for $\beta[6]$ :

$F_{m}\left(K r_{1}\right)-\frac{(m \bar{\beta})^{2}}{F_{m}\left(K r_{1}\right)}=\left(\frac{K r_{1}}{k r_{1}}\right)^{2} S_{m}\left(k r_{1}, k r_{0}\right)$,

where $\bar{\beta}=\beta / k, K^{2}=k^{2}-\beta^{2}$ (the usual waveguide equation) and $r_{0}$ is the radius to the bottom of the corrugation so that $\left(r_{0}-r_{1}\right)$ is the slot depth. Note this is the notation used by Olver et al. [5] for the inner and outer radii of the guide. $S_{m}(x, y)$ is given by the equation:

$S_{m}(x, y)=x \frac{J_{m}^{\prime}(x) Y_{m}(y)-J_{m}(y) Y_{m}^{\prime}(x)}{J_{m}(x) Y_{m}(y)-J_{m}(y) Y_{m}(x)}$.

For the hybrid modes the axial components of the electric and magnetic fields for the two possible orthogonal modes are defined to be:

$E_{z}(r, \phi)=a_{m} J_{m}(K r)\left(\begin{array}{c}\cos m \phi \\ \sin m \phi\end{array}\right)$

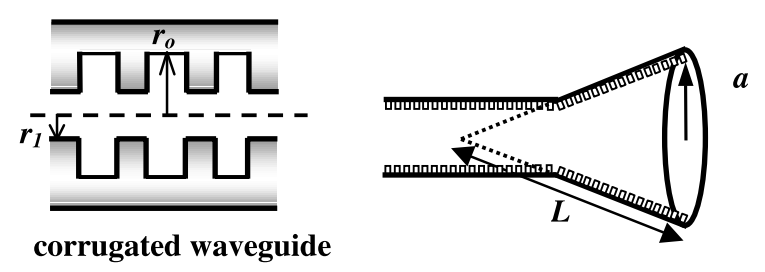

Fig. 3. Sketch of corrugated waveguide and horn defining inner radius of guide $r_{1}$, radius to bottom of slot $r_{0}$, slant length of horn $L$ and horn aperture radius $a$. 
and

$H_{z}(r, \phi)=a_{m} y_{0} \bar{\Lambda} J_{m}(K r)\left(\begin{array}{c}\sin m \phi \\ -\cos m \phi\end{array}\right)$

where $y_{0}$ is the admittance of free space and $\bar{\Lambda}$ is the hybrid factor (ratio of axial magnetic to electric field). The requirement that the $\phi$-component of the electric field be zero at the corrugations yields the following relationship between $\bar{\Lambda}$ and $\bar{\beta}$ :

$\bar{\Lambda}=-\frac{m \bar{\beta}}{F_{m}\left(K r_{1}\right)}$.

The cut-off condition $\beta=0$ yields either $F_{m}\left(K r_{1}\right)=$ 0 , for the EH modes which exhibit pure TE-type properties in the limit, or $F_{m}\left(K r_{1}\right)=S_{m}\left(k r_{1}, k r_{0}\right)$, for the HE modes which are pure TM in the limit. In the former case the boundary condition corresponds to that of a TE mode in a guide of radius $r_{1}$, while the latter corresponds to a TM mode in a uniform waveguide of radius $r_{0}$. The low-frequency cut-off values for several low-order modes are shown in Fig. 4. The cut-off depends on the ratio $r_{1} / r_{0}$ for those modes that are pure TM-type in the limit and is independent of $r_{1} / r_{0}$ for the TEtype.
As the frequency is increased for fixed values of $r_{1}$ and $r_{0}$ the high-frequency cut-off condition is eventually reached where $\bar{\beta}$ tends to $\infty$. Fig. 5 shows dispersion curves for modes of azimuthal order 1 and 2. As $k r_{1}$ increases from the lowfrequency cut-off, a balanced-hybrid condition is reached where $\bar{\Lambda}^{2}=1$. For high frequencies the $\mathrm{HE}_{11}$ mode is balanced hybrid when the corrugation depth approaches $\lambda / 4$. A fast wave to slow wave transition takes place at $\bar{\beta}=1$ (dashed line). Beyond this, in the slow-wave domain, a highfrequency cut-off eventually occurs where $\bar{\beta}$ tends to infinity and the mode terminates. Fig. 6 shows a plot of the high-frequency cut-off values for the $\mathrm{HE}_{n 1}$ and a selection of other modes.

Charts such as Figs. 4 and 6 can be used to determine the bandwidth of corrugated guide if certain modes are to be propagated. Within the surface impedance model, a mode is sustained with increasing frequency over the range in which the corrugation depth increases by slightly more than $\lambda / 2$. In the case of the high-frequency cut-offs, higher-order space harmonics have a non-negligible effect, and an error, depending on the mode and waveguide parameters, is to be expected for the surface impedance model predictions. Clarricoats

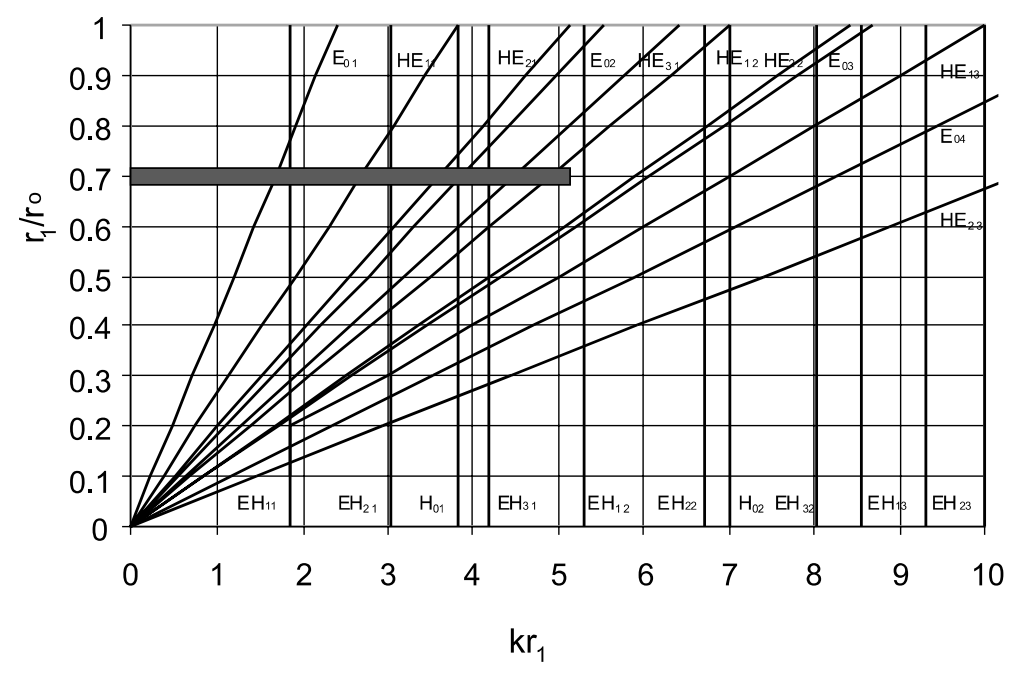

Fig. 4. Low-frequency cut-off hybrid mode chart where $k$ is the cut-off wave number, $r_{1}$ is the inner guide radius and $r_{0}$ is the radius to the bottom of the corrugation slots. For example, for $r_{1} / r_{0}=0.7$ and $k r_{1}=5.0$ the highest-order mode that can just propagate is the $\mathrm{HE}_{12}$ mode. 

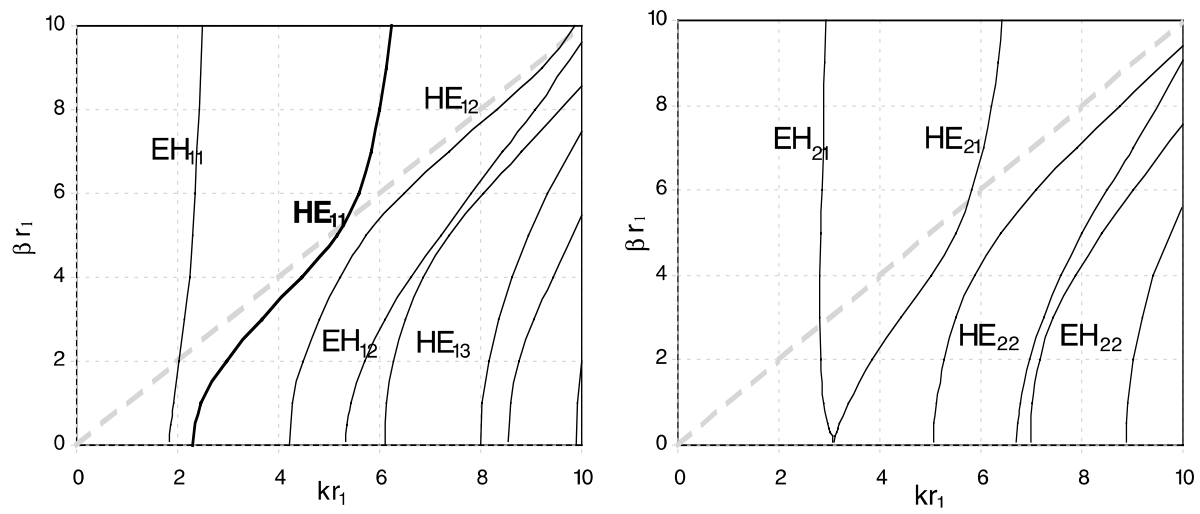

Fig. 5. Dispersion characteristics for modes of azimuthal order 1 and 2 in a corrugated waveguide with ratio of inner radius $r_{1}$ to outer radius to bottom of slot $r_{0}$ of $r_{1} / r_{0}=0.6$. $k$ is the free space wave number and $\beta$ is the guide wave number. For free space the dispersion curve is given by the dashed straight line as $k=\beta$.

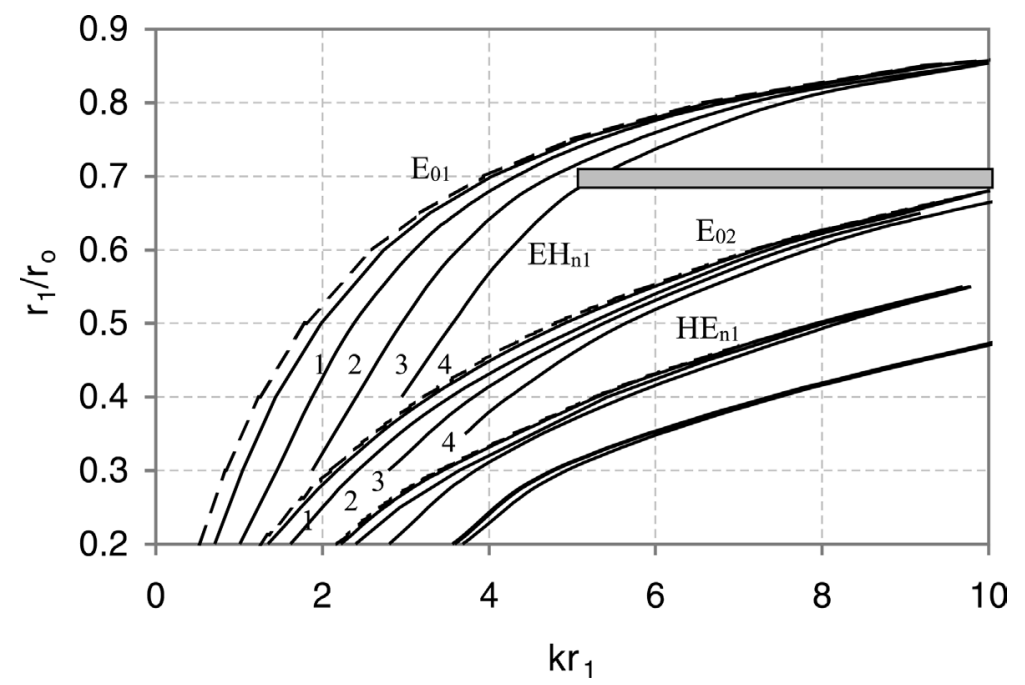

Fig. 6. High-frequency cut-off hybrid mode chart where $k$ is the high-frequency cut-off wavenumber, $r_{1}$ is the inner guide radius and $r_{0}$ is the radius to the bottom of the corrugation slots. 1, 2, 3, 4 refer to the $n$ in $\mathrm{HE}_{n 1}$ and $\mathrm{EH}_{n 1}$. Thus, for $r_{1} / r_{0}=0.7$ the fundamental $\mathrm{HE}_{11}$ mode is close to cut-off for $k r_{1} \approx 10.0$ while $\mathrm{EH}_{11}$ is cut off at $k r_{1} \approx 4.0$. (after Olver et al. [5]).

and Olver [6] show these to be of the order of $10 \%$.

As the waveguide flares out into the horn the hybrid modes develop a spherical wavefront with centre of curvature at the apex of the cone defined by the horn wall. The characteristics of the hybrid modes change in a complex but gradual way from the guide to the horn aperture in terms of the balance $\bar{A}$ between the TE and TM components (determined by relative strengths of $E_{z}$ and $H_{z}$ components). The normalized guide wavelength $\bar{\beta}$ also changes in a more complex way than is true for smooth walled horns as is clear from Fig. 5. The components of the electric fields of the two 
possible orthogonal $\mathrm{HE}_{n l} / \mathrm{EH}_{n l}$ hybrid modes at the horn aperture can be shown to be:

$$
\begin{aligned}
e_{r}= & E_{0}\left[(\bar{\Lambda}-\bar{\beta}) J_{n+1}\left(K_{n l} r / a\right)+(\bar{\Lambda}+\bar{\beta}) J_{n-1}\left(K_{n l} r / a\right)\right] \\
& \times\left(\begin{array}{c}
\cos (n \phi) \\
\sin (n \phi)
\end{array}\right) \exp \left(-\mathrm{j} k r^{2} / 2 L\right), \\
e_{\phi}= & E_{0}\left[(\bar{\Lambda}-\bar{\beta}) J_{n+1}\left(K_{n l} r / a\right)-(\bar{\Lambda}+\bar{\beta}) J_{n-1}\left(K_{n l} r / a\right)\right] \\
& \times\left(\begin{array}{c}
\sin (n \phi) \\
-\cos (n \phi)
\end{array}\right) \exp \left(-\mathrm{j} k r^{2} / 2 L\right),
\end{aligned}
$$

where $\bar{\beta}^{2}=1-(K / k a)^{2}$. If the corrugation depths are adjusted to be approximately equal to $\lambda / 4$ then the balanced-hybrid condition is satisfied and $\bar{\Lambda}=$ +1 (for $\mathrm{HE}_{n l}$ modes with $K_{n l}=p_{n-1, l}$ ) or $\bar{\Lambda}=-1$ (for $\mathrm{EH}_{n l}$ modes with $K_{n l}=p_{n+1, l}$ ), and it becomes true that the fields are zero at the edge of the aperture (e.g. modes shown in Fig. 7). The balanced-hybrid mode fields at the mouth of a horn re-expressed in terms of Cartesian unit vectors then becomes:

$$
\begin{aligned}
\mathbf{E}_{\mathrm{HE}_{n l}=} & E_{0} \frac{J_{n-1}\left(p_{n-1 l} r / a\right) \exp \left(-\mathrm{j} k r^{2} / 2 L\right)}{\sqrt{4 \pi a^{2}\left[J_{n}\left(p_{n-1 l}\right)\right]^{2}}} \\
& \times\left(\begin{array}{c}
\cos [(n-1) \phi] \mathbf{i}-\sin [(n-1) \phi] \mathbf{j} \\
\sin [(n-1) \phi] \mathbf{i}+\cos [(n-1) \phi] \mathbf{j}
\end{array}\right), \\
\mathbf{E}_{\mathrm{EH}_{n l}=} & E_{0} \frac{J_{n+1}\left(p_{n+1 l} r / a\right) \exp \left(-\mathrm{j} k r^{2} / 2 L\right)}{\sqrt{4 \pi a^{2}\left[J_{n}\left(p_{n+1 l}\right)\right]^{2}}} \\
& \times\left(\begin{array}{c}
\cos [(n+1) \phi] \mathbf{i}+\sin [(n+1) \phi] \mathbf{j} \\
\sin [(n+1) \phi] \mathbf{i}-\cos [(n+1) \phi] \mathbf{j}
\end{array}\right),
\end{aligned}
$$

where $a$ is the radius of the horn aperture, and $L$ is the slant length of the horn (see Fig. 3).

In general the horn will be many wavelengths in diameter and the far-field patterns can be assumed to be given by a simple Fourier transform relationship (paraxial limit). Thus, if the modes at the
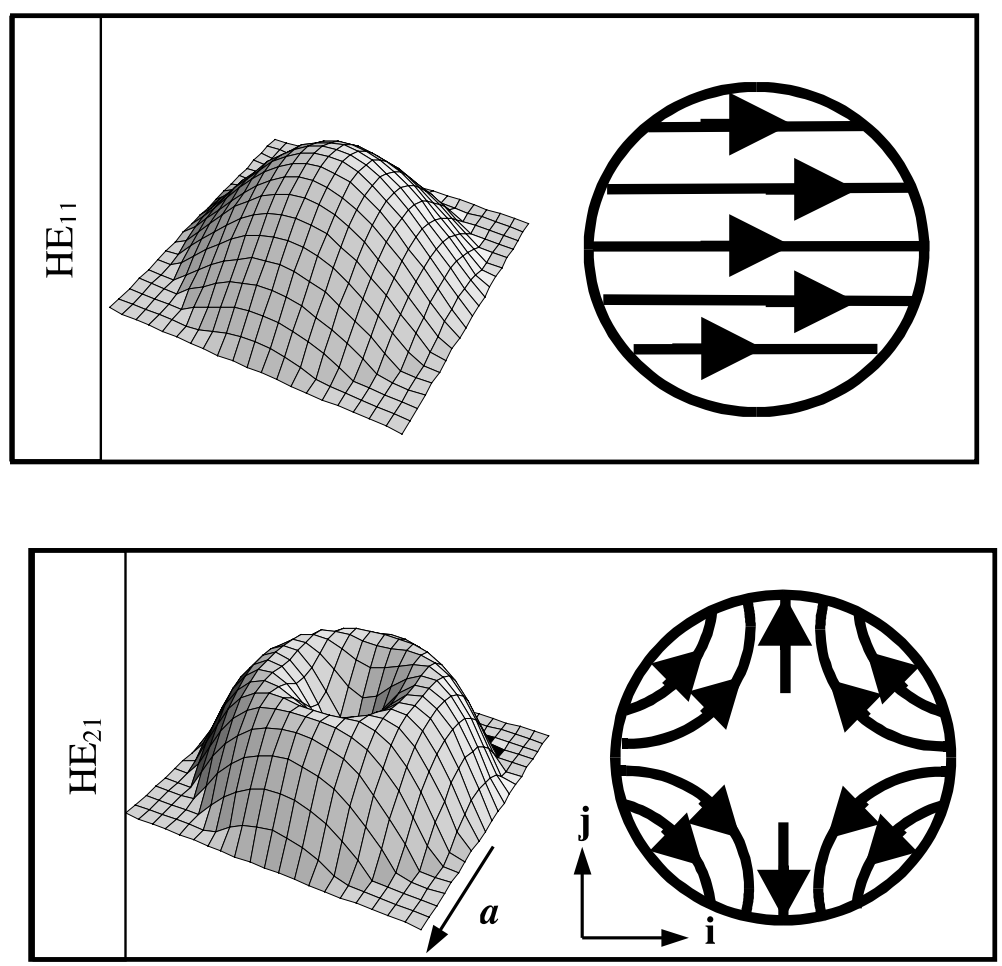

Fig. 7. Balanced hybrid modes $\mathrm{HE}_{11}$ and $\mathrm{HE}_{21}$ at horn aperture. 
horn aperture are balanced HE/EH modes then the radiation pattern is given by:

$$
\begin{aligned}
P_{\text {total }}(\theta)=\sum_{n l}^{\mathrm{HE}} & \left(\left\{\int_{0}^{a} J_{n-1}\left(p_{n-1 l} r / a\right) J_{n-1}(k r \sin \theta)\right.\right. \\
& \left.\times \exp \left(-\mathrm{i} k r^{2} / 2 L\right) r \mathrm{~d} r\right\}^{2} \\
& \left.\times\left(4 \pi a^{2}\left[J_{n}\left(p_{n-1 l}\right)\right]^{2}\right)^{-1}\right) \\
+ & \sum_{n l}^{\mathrm{EH}}\left(\left\{\int_{0}^{a} J_{n+1}\left(p_{n+1 l} r / a\right) J_{n+1}(k r \sin \theta)\right.\right. \\
& \left.\times \exp \left(-\mathrm{i} k r^{2} / 2 L\right) r \mathrm{~d} r\right\}^{2} \\
& \left.\times\left(4 \pi a^{2}\left[J_{n}\left(p_{n+1 l}\right)\right]^{2}\right)^{-1}\right) .
\end{aligned}
$$

The assumption here is that all modes carry equal power. This will be valid if the hybrid modes maintain their integrity and couple independently to the integrating cavity. In practice this means that the transition between the guide and the flared horn sections should be slow enough and smooth enough that scattering to higher-order hybrid modes does not occur at the horn-guide discontinuities. The horn aperture is wide enough that the effective impedance there is the same for all modes. In the next section we discuss the design of a multimoded horn using the ideas discussed in this and the previous sections.

\section{The design of multi-moded corrugated horns}

The waveguide filter at the back of the horn determines the number of modes that can propagate. If we assume that there are many corrugations per wavelength across the band of interest $(>3)$ then we can use the hybrid mode model as an approximation. Some basic points about the corrugated filter should be noted. A bandpass filter generally defines the bandwidth of multi-moded horns (typically $\Delta \lambda / \lambda$ of $1 / 4$ to $1 / 3$ ). However, for very wide-band operation it may be important to know over what range of wavelengths the funda- mental $\mathrm{HE}_{11}$ mode can propagate. At the longwavelength (low frequency) cut-off point the $\mathrm{HE}_{11}$ mode behaves like a $\mathrm{TM}_{11}$ mode in a guide defined by the radius $r_{0}$ to the bottom of the slot. Thus, the $\mathrm{HE}_{11}$ cut-off wavelength $\lambda_{\mathrm{c}}$ is given by: $\lambda_{\mathrm{c}}=$ $2 \pi r_{0} / 3.83171$. Note that the $\mathrm{EH}_{11}$ and $\mathrm{E}_{01}$ modes have longer wavelength cut-offs (behaving like $\mathrm{TE}_{11}$ and $\mathrm{TM}_{01}$ modes in a smooth guide of radius $r_{1}$ ), but will not propagate unless the corrugations are very shallow, as is clear from Fig. 4 . It is also important that the $\mathrm{HE}_{11}$ mode not be close to its high-frequency cut-off at the operating wavelength, otherwise the horn will lose on-axis gain and a hole will appear in the centre of the radiation pattern. This means in effect that the ratio of $r_{1} / r_{0}$ must not be too small, or in other words the slots must not be too deep $(<\lambda)$, as is clear from Fig. 6.

As an example, if optimizing operation for wavelength $\lambda$ we choose the waveguide filter such that $r_{1} / r_{0}=0.7$ and $k r_{1}=5.02$ (or $r_{1}=0.8 \lambda$ ), then the slot depth is $0.34 \lambda$ (i.e. approximately $\lambda / 3$ ) and $r_{0}=1.14 \lambda$. Using Figs. 4 and 6 we predict that the modes which can propagate are $\mathrm{H}_{01}, \mathrm{HE}_{11}, \mathrm{HE}_{12}$, $\mathrm{HE}_{21}, \mathrm{HE}_{31}$ and $\mathrm{E}_{02}$. In general the $\mathrm{E}_{01}$ and the $\mathrm{EH}_{n 1}$ modes are evanescent as is clear from Fig. 6 and the $\mathrm{HE}_{11}$ long-wavelength cut-off is at $\lambda_{\mathrm{c}}=$ $1.86 \lambda$, where $\lambda$ is the design frequency for which the performance is optimized. Once the modes determined by the waveguide filter are known we can specify the relevant modal fields at the horn aperture as in Section 3. The frequency at which the best sidelobe rejection is required essentially determines the slot depths near the horn aperture. As the horn flares out the slot depths should be adjusted to $\lambda / 4$ [6]. As a practical example we have chosen the aperture radius $a=4.1 \mathrm{~mm}$ and slant length $L=28 \mathrm{~mm}$ with the waveguide filter section defined as above. The predicted far-field radiation pattern is shown in Fig. 8 for the case of $\lambda=550$ $\mu \mathrm{m}$ using the hybrid mode model, with the contributions of the component modes shown in Fig. 9. However, because of the approximations inherent in the approach we do not expect high accuracy for the beam pattern.

The modal-matching scattering model allows us to compute the radiation pattern of a horn more accurately including the real corrugations. Thus, 


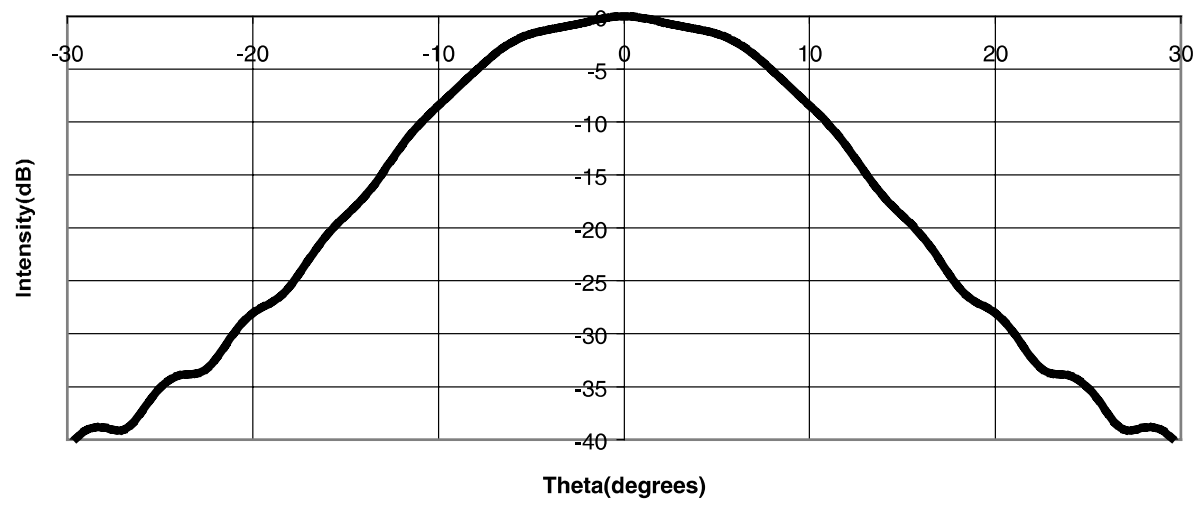

Fig. 8. Radiation pattern of conical horn. Hybrid mode model with contributing modes: $\mathrm{HE}_{11}, \mathrm{HE}_{12}, \mathrm{HE}_{21}, \mathrm{HE}_{31}, \mathrm{EH}_{12}, \mathrm{H}_{01}$ and $\mathrm{E}_{02}$. For the horn in question $\lambda=550 \mu \mathrm{m}, a=4.1 \mathrm{~mm}$ and $L=28 \mathrm{~mm}$.

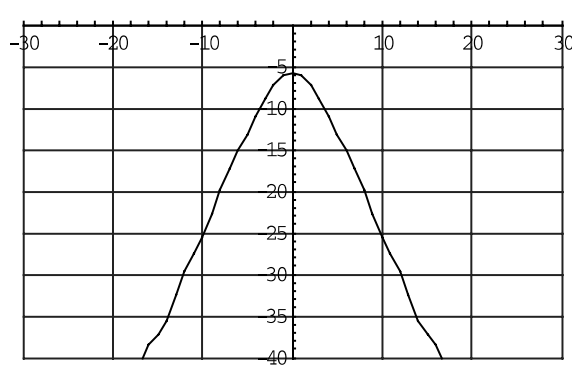

$H E_{11}$

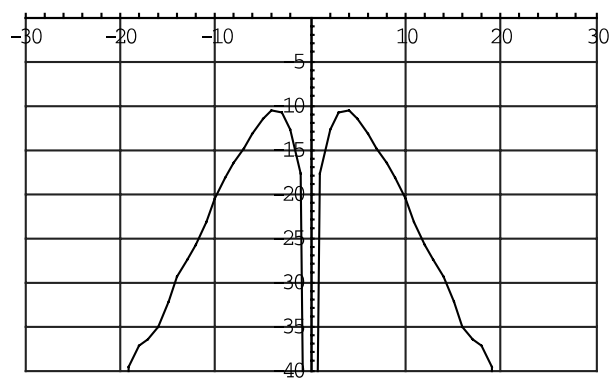

$H E_{21}, H_{01} \& E_{02}$

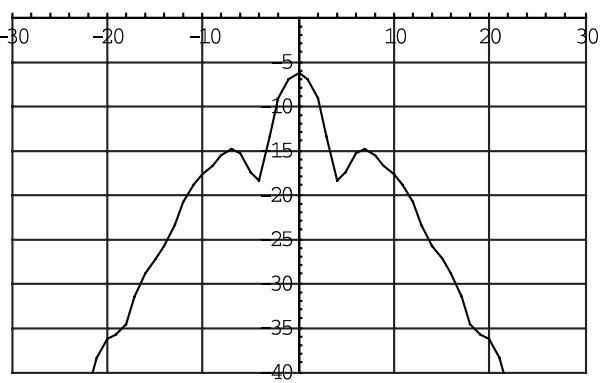

$H E_{12}$

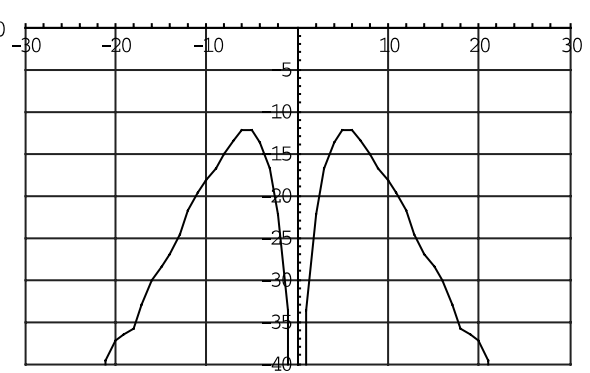

$H E_{31} \& E H_{12}$.

Fig. 9. Contributions of the $\mathrm{HE}_{11}, \mathrm{HE}_{12}, \mathrm{HE}_{21}, \mathrm{HE}_{31}, \mathrm{EH}_{12}, \mathrm{H}_{01}$ and $\mathrm{E}_{02}$ to the beam pattern shown above in Fig. 8. Same scales as Fig. 8.

we can investigate the effects of varying the corrugation pitch and the details of the transition section over which the corrugation depths are adjusted between the filter section and the horn aperture. In Fig. 10 we show the radiation patterns for the same horn as referred to above ( $a=4.1$ $\mathrm{mm}, L=28 \mathrm{~mm}$ ) but for corrugation pitches of $\lambda$ (very crude corrugations) and $\lambda / 4$. In two cases a short linear transition section near the horn throat was used to step the corrugation depths from $\lambda / 3$ 


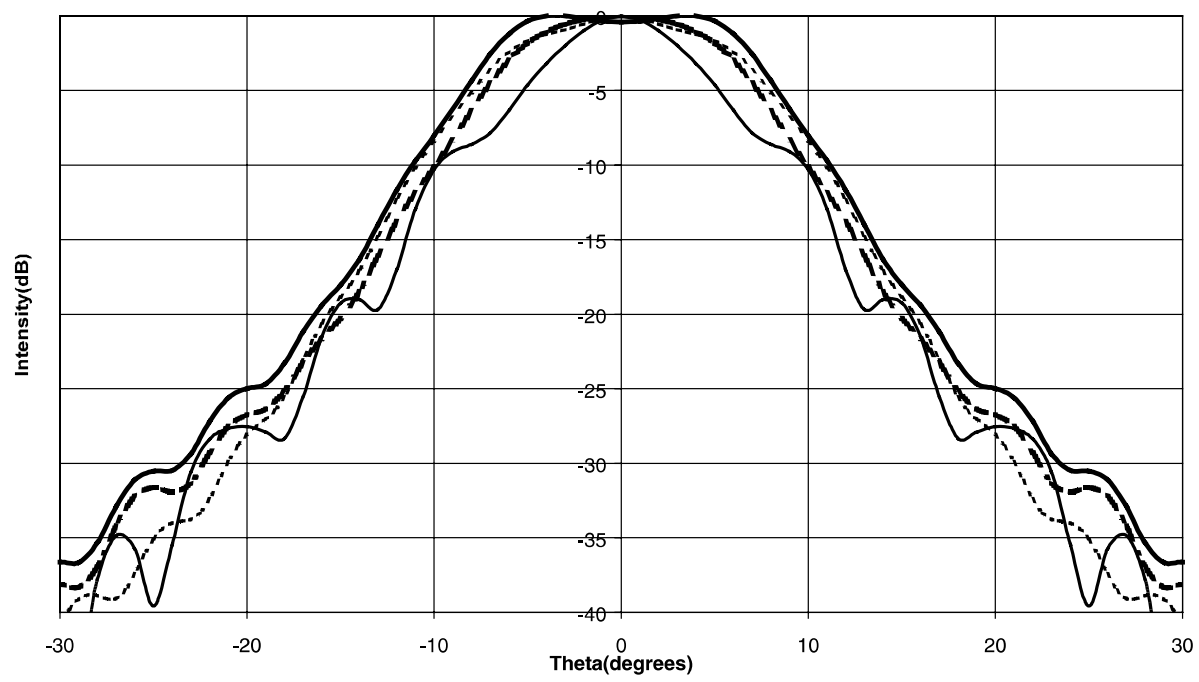

Fig. 10. Modal-matching model. Effect of varying pitch of corrugations and transition section length on radiation pattern of conical horn, (i) thick solid line: corrugation pitch of $\lambda / 4$, short transition; (ii) thin solid line: corrugation pitch of $\lambda$, short transition; (iii) thick dashed corrugation pitch of $\lambda / 4$, transition over length of horn. For comparison the thin dashed line shows the surface impedance model.

to $\lambda / 4$. If the transition is extended over the full length of the horn a significant change in the radiation pattern is noticeable, as is clear in Fig. 10 for the second $\lambda / 4$ pitch example. Superimposed on the same plot is radiation pattern predicted using the hybrid mode model. Clearly, given the level of variation possible in the radiation patterns the hybrid mode result must ultimately be accepted as only approximate.

It is also interesting to consider the effect of profiling the horn to obtain a narrower radiation pattern (mimic a much longer conical horn). The simple hybrid mode model is inadequate for determining the aperture fields of such a system ac- curately as there will be some hybrid modal scattering through the profiled section of the horn. In such a case a scattering matrix modal-matching approach is clearly necessary. We present as an example a profiled horn where the shape has a sine squared taper (see Fig. 11). The horn aperture size is as for the previous example $(a=4.1 \mathrm{~mm})$. The predicted beam pattern using the modal-matching approach is shown in Fig. 12. The pattern clearly has higher gain (narrower far-field FWHM for example).

If a single mode horn is used to feed an antenna then clearly the focal point should coincide with horn phase centre in order to optimize the gain of

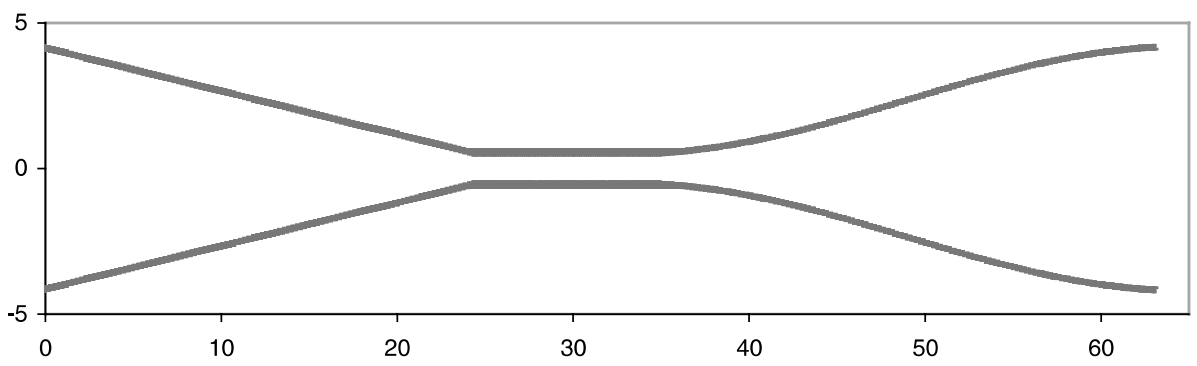

Fig. 11. Profiled overmoded corrugated horn also showing flared backsection. All dimensions are in millimetres. 


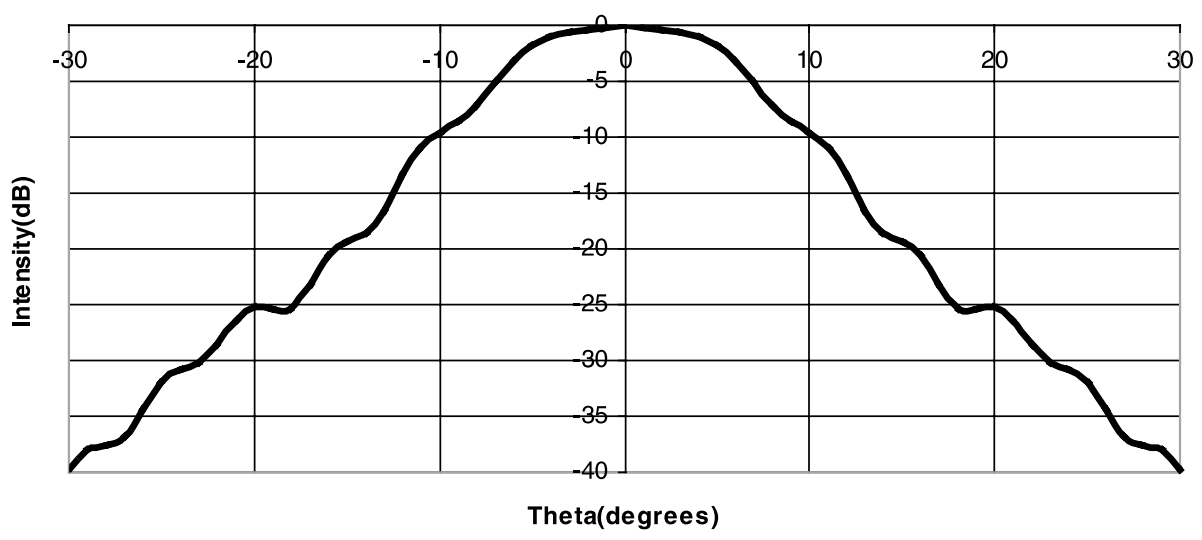

Fig. 12. Radiation pattern profiled horn with aperture radius $a=4 \mathrm{~mm}, \lambda=550 \mu \mathrm{m}$ with same corrugations as horn described in Fig. 10(i).

the system. In the case of a multi-moded horn the phase centre, as such, is an ambiguous term and, in general, each independently propagating mode will have a different phase centre location. A more meaningful and useful concept is that of virtual beam waist (the plane at which the width of the beam is narrowest when propagated backwards from the horn aperture as though in free space). The optimum resolution with an antenna is then obtained when the horn beam waist is coincident with the focal plane, since a diffraction limited image of the waist is formed on the sky. The location of the virtual horn beam waist involves plotting the near-field beam at various planes by summing in quadrature the component independent mode beam patterns after application of the appropriate Fresnel transformations.

\section{Conclusions}

In this paper we have discussed two different theoretical approaches to modelling corrugated horn-filter configurations. One approach is based on mode matching regarding the horn to be made up of consecutive smooth walled waveguide segments and taking into account the consequent scattering of power between the pure TE and TM cylindrical waveguide modes. The second hybrid mode approach approximates the corrugated waveguide and horn walls by a uniform impedance surface and the propagation is defined in terms of hybrid HE and EH modes. The model assumes that this effective surface impedance varies only slowly and takes no account of any scattering that may occur within the horn or waveguide. It is thus more approximate than the mode-matching model, which actually incorporates all of the scattering that occurs at the individual corrugations in the guide and horn. The mode-matching model must be assumed therefore to be accurate and reliable, although, of course, ultimately limited by the finite number of modes included in the calculations and any numerical instabilities that occur in using a cascading process with a large number of steps. We have found no particular problems with such instabilities with typical few-moded corrugated horns when the number of modes in the calculation was kept to a minimum (10-20 modes), consistent with a convergent result.

The mode-matching model has the disadvantage of being computationally intensive. By comparison the hybrid mode model is very fast to implement and particularly useful in predicting the number of modes that will be transmitted by the waveguide filter section. Reasonably good agreement between the two models in terms of predicting the radiation pattern of conical corrugated horns was obtained, although there was some dependence of the patterns on the corrugation taper details. Furthermore, if the horns are profiled to enhance the beam quality then the simple hybrid 
mode model is inadequate for determining the radiation pattern and the scattering matrix modalmatching approach is necessary.

Corrugated overmoded horns are planned for use as incoherent detector feeds on forthcoming space missions. For example, the 550 and $850 \mathrm{GHz}$ channels on the High-Frequency Instrument (HFI) of the PLANCK Surveyor will use overmoded horn antennas as the detector feeds to achieve the highest coupling efficiency to the cosmic microwave background for cases where the resolution is not diffraction limited. It also is envisaged that few-moded bolometric feed horns will be used on the SPIRE Instrument on the Far-IR and Submillimeter Space Telescope (FIRST). The models described in this paper will be used in the design of these systems.

Ultimately, the verification of the models and their implementation in software depends on a well designed programme of experiments on overmoded horn antennas. This is being planned as part of the development of the HFI instrument for PLANCK. Preliminary results are however available for a prototype corrugated HFI horn, designed to be single moded, but which was operated at a higher frequency so that an overmoded response takes place. Comparison with the measurements indicates good agreement between experiment and the predictions of the two models. More systematic measurements of horn antennas actually designed to be overmoded is planned for the near future.

One limitation of the approach is the assumption of good coupling of the waveguide to the integrating cavity. This can be achieved through flaring of the guide, but it would be clearly ad- vantageous to be able to model the coupling of an unflared waveguide directly to a cylindrical cavity in which an absorber is placed. Future development of the technique will concentrate on the integrating cavity, so that the optimum location of the absorber can be determined. Thus, any reflections at the guide cavity interface can be reduced to a minimum and the coupling to the incoming radiation from the far-IR source maximized.

\section{Acknowledgements}

The authors would like to acknowledge the support of Enterprise Ireland in this research programme.

\section{References}

[1] B. Maffei, et al., Corrugated Gaussian back-to-back horns for cosmic microwave background continuum receivers, IEE Antenna Symposium 2000, QMW, London, April 2000.

[2] S.E. Church, et al., A compact high-efficiency feed structure for cosmic microwave background astronomy at millimetre wavelengths, 30th ESLAB Symposium on Submm and Far-IR Space Instrumentation, ESTEC, Nordwijk, The Netherlands, 1996.

[3] J.A. Murphy, R. Padman, Radiation patterns of few moded horns and condensing lightpipes, Infrared Physics 31 (1991) 291.

[4] R. Padman, J.A. Murphy, Radiation patterns of scalar horns, Infrared Physics 31 (1991) 441.

[5] A.D. Olver, P.J.B. Clarricoats, A.A. Kishk, L. Shafai, Microwave Horns and Feeds, IEEE Press, 1994.

[6] P.J.B. Clarricoats, A.D. Olver, Corrugated Horns for Microwave Antennas, Peter Peregrinus (for IEE), 1984.

[7] S. Ramo, J.R. Whinnery, T. van Duzer, Fields and Waves in Communication Electronics, second ed., Wiley, 1984. 